\title{
Amistad y reconocimiento. Sobre la philia aristotélica. Lo que Aristóteles vio y Hegel pasó por alto*
}

\section{Friendship and recognition. On aristotelian philia. What Aristotle saw and Hegel overlooked}

\author{
MIGUEL MARTÍ SÁNCHEZ \\ Universidad de Navarra
}

Recibido: 14/06/2017 Aceptado: 22/’7/2017

\begin{abstract}
RESUMEN
La definición aristotélica del hombre como animal político (zoon politikon) no está exenta de posibles interpretaciones, entre ellas la de que sólo dentro de una polis puede el ser humano ser feliz. A partir del caso de la philia tal y como aparece, sobre todo, en los libros VIII y IX de la Ética a Nicómaco y en relación con la figura del spoudaios que aparece también en ambos libros, propongo una lectura 'política' de la Ética a Nicómaco. Entiendo por 'lectura política' una lectura en la cual el hilo conductor y motivo fundamental de la ética es, antes
\end{abstract}

* Este artículo fue presentado como ponencia invitada en la II Jornada del Seminario Permanente «Grecia y Alemania»: El legado de Grecia en la Filosofía Alemana en la Universidad de Málaga el 12 de mayo de 2017. Quiero agradecer a los profesores Juan José Padial, Javier Hernández Pacheco, Jesús de Garay, Cristóbal Holzapfel y Manuel Barrios sus valiosas observaciones. Por último quiero transmitir también mi agradecimiento a Sergio Clavero por su ayuda a la hora de pulir la interpretación de Hegel que se hace aquí; no obstante, la exégesis que se hace, con los errores que pueda haber, corre por entero a mi cargo.

(C) Contrastes. Revista Internacional de Filosofia, vol. XXII-N² (2017), pp. 37-49. ISSN: 1136-4076 Departamento de Filosofía, Universidad de Málaga, Facultad de Filosofía y Letras Campus de Teatinos, E-29071 Málaga (España) 
que la investigación acerca de las condiciones necesarias para lograr la felicidad individual, una que versa sobre el carácter idóneo del buen hombre (spoudaios) y del buen ciudadano. En segundo lugar, establezco algunos paralelismos entre esta lectura y la interpretación hegeliana de algunas ideas aristotélicas. Concluyo que la omisión de un tratamiento pormenorizado de la philia en la ética-política de Hegel implica una diferencia fundamental en el abordaje que ambos autores hacen de la filosofía práctica.

\author{
PALABRAS CLAVE \\ PHILIA, AMISTAD, POLIS, EUDAIMONIA, SPOUDAIOS, VIRTUDES, ESTADO.
}

\begin{abstract}
The Aristotelian definition of man as a political animal (zoon politikon) is not exempt from possible interpretations, including that only within a polis can the human being be happy. From the case of the philia as it appear, above all, in books VIII and IX of Nicomachean Ethics and in relation to the figure of spoudaios that appears also in both books, I propose a 'political' reading of Nicomachean Ethics. I understand by 'political reading' a conception in which the guiding thread and fundamental motive of ethics is, rather than research into the necessary conditions to attain individual happiness, one concerning with the ideal character of the good man (spoudaios) and of the good citizen. Secondly, I make some parallels between this reading and the Hegelian interpretation of some Aristotelian ideas. I conclude that the omission of a detailed treatment of the philia in Hegel's ethics-politics implies a fundamental difference in the approach that both authors make of the practical philosophy.
\end{abstract}

KEYWORDS

PHILIA, FRIENDSHIP, POLIS, EUDAIMONIA, SPOUDAIOS, VIRTUES, STATE.

\title{
I. INTRODUCCIÓN
}

LA INVESTIGACIÓN SOBRE LA VIRTUD de la philia o amistad ocupa un lugar principal en la Ética a Nicómaco y en otros tratados de la filosofía práctica aristotélica como la Política y la Retórica. Aristóteles considera que la posesión de dicha disposición habitual tiene una importancia capital tanto para la felicidad del individuo como para la de la polis; mientras la philia teleia o amistad en sentido pleno forma parte del carácter de la persona virtuosa o spoudaios, en el caso de la polis es la philia politike el tipo de relación intersubjetiva a la que debe aspirar la mejor constitución o régimen político. Así como para Aristóteles la ética tiene una función protréptica además de científica, es decir, pretende, por así decir, no quedarse en mera teoría sino fomentar la práctica de las virtudes; por su parte la política se encarga de averiguar cómo proceder para que la polis sea un lugar adecuado para ese ejercicio de las excelencias humanas. En este sentido para Aristóteles hay una conexión esencial entre el ideal de la excelencia humana y qué tipo de polis es la que actualiza tal ideal del mejor modo posible. A su juicio la mejor polis posible es aquella en la que 
se promueve la amistad cívica o philia politike, y no meramente relaciones de justicia - p.e. contractuales- o afectivas - p.e. familiares-.

A pesar de que algunos intérpretes de la filosofía práctica de Hegel han intentado ver una conexión entre ciertos aspectos de dicha filosofía -p.e. la importancia concedida al reconocimiento recíproco- y la aristotélica, lo cierto es que las diferencias entre ellas son casi insalvables. Esto se manifiesta con claridad en la facilidad con la que Hegel despacha la amistad en su dimensión socio-política. Ahora bien ¿cuáles son las razones que llevaron a Hegel a considerar la amistad como algo prácticamente irrelevante para la política?

La tesis que sugiero en mi intervención es que esto ocurre porque Hegel pasa por alto una de las intuiciones fundamentales de la filosofía práctica aristotélica, a saber: que es la polis la que está al servicio del ejercicio de las virtudes y no al revés. Esto se muestra con claridad en el caso omiso que hace Hegel tanto de la amistad como de la amistad cívica. Para el filósofo alemán en la medida en que es el Estado la institución que hace posible la libertad máxima de los individuos el resto de manifestaciones o momentos de lo político -el derecho y la moralidad- deben estarle subordinados, esto incluye también el carácter y conciencia de los ciudadanos así como las relaciones amistosas que establezcan entre ellos.

Como según Hegel la amistad y el amor son meros sentimientos -de hecho solo este último forma parte de la eticidad en la forma de lo natural dentro de la familia-deben ser superados en el proceso dialéctico que conduce al Estado como figura suprema de la eticidad. Dicha comprensión del amor y la amistad está teñida de las teorías románticas tan en boga en la época de Hegel. Esta concepción sentimental de la amistad y el acercamiento dialéctico a la realidad política alejan a Hegel de Aristóteles, para quien la amistad es una virtud y cuya comprensión de lo político es teleológica.

El orden de mi exposición será el siguiente: Primero, desarrollo brevemente la teoría de la amistad de Aristóteles. En ella se muestra que la amistad no es solo una virtud del individuo sino también una virtud política. Con el fin de aclarar este punto haré algunas observaciones acerca de cómo se relacionan ética y política para Aristóteles. En segundo lugar, resumiré la concepción hegeliana de la amistad como mero sentimiento, y daré algunas razones que explican por qué Hegel no concedió ninguna importancia a la amistad en su concepción de la política. Y por último señalaré las similitudes y diferencias entre la propuesta aristotélica y la hegeliana.

\section{LA PHILÍA ES POLISÉMICA}

Como ocurre con muchos conceptos que Aristóteles investiga también la philia o amistad en un término que posee varios significados o como diría el Estagirita «se dice de muchas maneras» (legetai pollajos). Con el fin de aclarar 
su significado Aristóteles diferencia entre tipos de amistad y establece una clasificación a lo largo de los libros VIII y IX de la EN. En estos se distingue entre: amistad (1) por interés o utilidad; (2) por placer; y aquella que es (3) perfecta o plena ( $p$ hilia teleia) porque está cimentada en el carácter excelente propio y el del amigo; y fundada sobre esta última se añade una cuarta que Aristóteles menciona en la Política, (4) la amistad cívica o philia politike.

Esta tipología descansa sobre el principio siguiente: existe una relación proporcional entre el tipo de amistad y el tipo de bien que los amigos quieren para cada uno (ENVIII 3, 1156a 9-10), por ejemplo, si solo el afecto sensible es la causa de una amistad, entonces se trata de una amistad de tipo (2); en cambio, si el carácter excelente del amigo es lo que reconozco en él como motivo de la amistad, entonces se trata de la amistad de tipo (3).

En los libros VIII y IX de la $E N$ concluye Aristóteles que la amistad en sentido pleno es una excelencia del carácter en forma de hábito (hexis) o disposición habitual y por lo tanto que es permanente. ${ }^{1}$ No es, por tanto, una pasión o un acto, es decir no pertenece por naturaleza a la naturaleza del hombre, sino que implica una decisión deliberada y el ejercicio continuado de acciones buenas realizadas por mor del bien mismo. Como es sabido, la adquisición de dichas aretai -entre las que se encuentra la amistad- y en definitiva de un ethos virtuoso se realiza a través de la educación y formación del carácter. En el caso concreto de la philia el sujeto portador de la virtud de la amistad se convierte en amigo, o mejor se hace capaz de ser amigo y de tener amigos. De ahí que a juicio de Aristóteles solo el hombre de carácter excelente (spoudaios) es un verdadero philos o amigo.

Aristóteles establece como significado focal de philia aquella que decíamos fundada en el carácter excelente, es decir la tipo (3) y en consecuencia también la (4). ${ }^{2}$

Para que se pueda decir con exactitud que existe tal tipo de amistad entre dos personas son necesarias, según Aristóteles, las siguientes condiciones:

(a) reciprocidad (en antipeponthosi), es decir, uno y otro son amigos.

(b) reconocimiento [me lanthánousan], es decir, uno y otro saben de la amistad que el otro les profesa.

(c) benevolencia (querer el bien del otro) (eunoia).

(d) carácter excelente (spoudaios).

(e) convivencia en comunidad o concordia (homonoia), es decir, pasar tiempo juntos.

1 «La amistad fundada en el carácter y que se busca por sí misma es permanente» ( $\dot{\eta} \delta \grave{\varepsilon}$

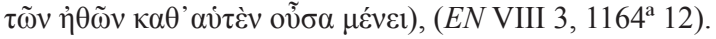

2 Se puede consultar el estudio de Cooper 1980, pp.301-340, al que nos referimos en la bibliografía para los que quieran saber más de los otros tipos de amistad. 
(f) recto amor a uno mismo (philautía), si él no es posible querer bien el bien del prójimo.

De este tipo de amistad ofrece Aristóteles una definición en la Retórica: «Sea pues amar (philein) querer para alguien lo que se considera bueno, en interés suyo, y no en el nuestro, y estar dispuesto a llevarlo a efecto en la medida de nuestras fuerzas. Y amigo es el que ama y es correspondido en su amor. Y creen ser amigos los que creen encontrarse en esa disposición mutua» (Ret., II 4, 1380b35-1381a3). Y en la $E N$ especifica que: «La amistad perfecta es la de los hombres buenos e iguales en virtud; porque estos quieren el bien el uno del otro en cuanto son buenos, y son buenos en sí mismos; y los que quieren el bien de sus amigos por causa de estos, son los mejores amigos, puesto que es por su propia índole por lo que tienen esos sentimientos y no por accidente; de modo que su amistad permanece mientras son buenos, y la virtud es una cosa permanente» (EN VIII 4, 1156b 7-12).

En resumen, la philia teleia es una disposición habitual del hombre excelente o bueno que se actualiza en el modo de querer el bien de otra persona y las razones por las que quiere ese bien para ella.

\section{LA PHILÍA ES VIRTUD POLÍTICA}

La philia es según Aristóteles una virtud social o política porque regula o configura las relaciones de unos sujetos con otros, y debe ser promovida por el gobierno de la polis. Por ejemplo, dice Aristóteles que: «La amistad mantiene unidas a las ciudades, y que los legisladores consagran más esfuerzos a ella

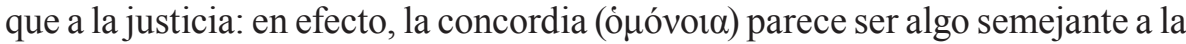
amistad, y es a ella a lo que más aspiran, mientras que lo que con más empeño procuran expulsar es la discordia, que es enemistad» (EN VIII 4, 1155 23-25).

La importancia de la polis como ámbito de desarrollo del carácter del ser humano fue reconocido por Aristóteles. ${ }^{3}$ De hecho esto se traduce de manera concreta en la relación esencial que este traza entre ética y política, por ejemplo al final de su Ética a Nicómaco. Aunque la manera de verificar dicha relación varía según los intérpretes, ${ }^{4}$ existe cierto consenso en un punto: Aristóteles

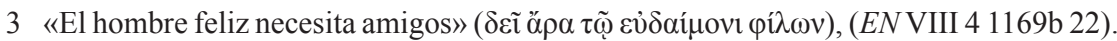

4 Como afirma Barker en la introducción a su célebre comentario de la Política de Aristóteles en cuatro volúmenes: «Aristóteles, después de haber tratado la moralidad en sus Éticas como una 'disposición' del individuo, en la Política se encarga de la moralidad como 'forma de vida' o sistema organizado del comportamiento, que funciona en una comunidad política que produce al mismo tiempo dicha 'disposición' mediante el gobierno, las leyes y la educación, y se ocupa con energía de que un círculo amplio de personas, metidos en una red de relaciones, puedan ejercitar dicha disposición y respirar esa forma de vida», Barker, 1968, p. xxxv. Esta traducción así como las que en adelante son mías a no ser que se indique lo contrario. 
mantiene que es la polis la que está al servicio del ejercicio de las virtudes y no al revés. Con el fin de explicar este principio quisiera traer a colación la exégesis de dos filósofos políticos conocidos por su neoaristotelismo, a saber: Eric Voegelin y de Leo Strauss.

Según Voegelin la ciencia de los asuntos humanos -tal y como Aristóteles

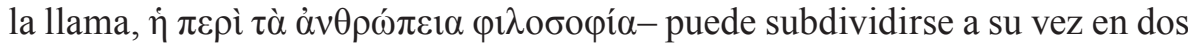
«ciencias especiales», a saber: la ciencia de las excelencias humanas por un lado, y la ciencia legislativa -en el sentido de nomos- por otro lado. ${ }^{5}$ Ahora bien, este esquema de la ciencia de los asuntos humanos no hace sino recoger lo que Aristóteles considera como dos momentos necesarios de la existencia humana, a saber: la formación del carácter de la persona individual y las instituciones necesarias para que tal formación sea posible. Esto tiene una consecuencia concreta para el estudio de los asuntos humanos en su dimensión política, a saber: del mismo modo que la felicidad individual debe ser estudiada por el investigador de asuntos éticos, así el investigador de los asuntos políticos comparte con el primero la importancia de la eudaimonia y añade a esta perspectiva la función de que esta no sea solo individual sino colectiva, es decir, política.

A juicio de Voegelin las nociones que conectan ambos tratados -a los que habría que añadir pasajes significativos de la Retórica- son las de philia, eudaimonia y spoudaios. Considera que el spoudaios o persona de carácter excelente es la figura que integra de manera más completa el ideal que es necesario realizar para que no sólo el sujeto individual actualice y desarrolle su naturaleza de la manera más excelente posible, sino que dicho crecimiento redunde en beneficio de toda la comunidad. Pues solo el spoudaios es capaz de amigos en sentido pleno (teleia), ya que este no solo actualiza su ser individual sino que en la medida en que cultiva su carácter de una determinada manera y en una dirección política, promueve también la felicidad de la polis. ${ }^{6}$

Del mismo modo que para Aristóteles no es posible la felicidad individual sin polis y una constitución o régimen ( politeia) adecuado, tampoco es posible la eudaimonia de la polis sin que el carácter de sus ciudadanos sea excelente (spoudaios). Por lo tanto, la función de la ética en cuanto ciencia no es sólo de investigación sino que también tiene carácter protréptico, es decir, exhorta y promueve la práctica de la virtud como el fin al que debe aspirar cualquier hombre y cualquier ciudadano. Aquí surge de nuevo la relación intrínseca entre

5 Afirma que: «el spoudaios es el hombre que realiza al máximo las potencialidades de la naturaleza humana, que formó su carácter en la realización habitual de las virtudes éticas y dianoéticas, el hombre que en su más completo grado de desarrollo es capaz de bios theoretikos. Por lo tanto, la ciencia de la ética en el sentido aristotélico es un estudio del tipo del spoudaios, Voegelin 1957, p. 83.

6 Dice Voegelin: «la philía aristotélica, el núcleo experiencial de verdadera comunidad entre hombres buenos», Voegelin 1957, p. 85. 
spoudaios y philia, a saber: con la noción de philia politike, como aquello a lo que la mejor constitución o el mejor régimen (aristen politea) deben aspirar.

Aristóteles afirma que es la polis la que está subordinada a la adquisición y práctica de las virtudes por parte del hombre y no al revés. A mi juicio tiene razón Strauss cuando afirma en su obra La Ciudad y el hombre que: «las virtudes morales no pueden entenderse como algo que está en función de la polis sino que más bien la polis debe entenderse como algo en función de la práctica de las virtudes morales» (Strauss 1978, p. 27).

Hemos visto hasta ahora que Aristóteles considera la amistad como un hábito moral deliberado y bueno, es decir, que dispone el carácter de manera deliberada (racional) a realizar lo bueno para con el prójimo y especialmente con el que asemeja a uno mismo, por lo tanto además de permanente es directamente social o política. Esta última dimensión se muestra en el interés de Aristóteles por conectar las investigaciones de ética con aquellas de carácter político, en concreto en la importancia que concede a la philia y la philia politike como integrantes del carácter tanto del virtuoso como del buen ciudadano (spoudaios), y como fin que deben perseguir las leyes que introduzca el gobernante de la polis.

\section{De Aristóteles a Hegel}

Sorprende prima facie que el aristotélico Hegel pase por encima de una doctrina que ocupa dos libros completos del tratado de moral más celebre de Aristóteles. ¿Qué razones pueden haber llevado a Hegel a concederle tan poca importancia a la amistad en su filosofía práctica? Tanto Peperzak como Williams afirman que la ausencia o presencia respectivamente de la amistad en sentido aristotélico lleva aparejadas consecuencias importantes para la filosofía práctica de Hegel. No obstante, considero que para responder a la pregunta antes formulada es necesario responder antes a otras dos: ¿Con qué noción de 'amor' y 'amistad' se encuentra Hegel y qué dice sobre ella? Y en segundo lugar, ¿qué dice sobre la relación entre la práctica de las virtudes y la necesidad de la polis para dicho ejercicio?

Brevemente podríamos resumir las indagaciones de Hegel en este punto de la siguiente manera. En las pocas ocasiones que Hegel habla de la amistad (Freundschaft) esta es definida como sentimiento (Empfindung) y perteneciente al ámbito de la moralidad y no de la eticidad. ${ }^{7}$ Por su parte el amor (Liebe) se

7 «Lo que propiamente llamamos voluntad (Willen) contiene en su interior los dos momentos precedentes. En un primer momento el yo es, en cuanto tal, pura actividad, la universalidad que permanece consigo misma; pero esta universalidad se determina y al hacerlo no queda consigo, sino que se pone como otro y deja de ser universal. Lo tercero es ahora que en su limitación, en ese otro, esté consigo misma, que al determinarse permanezca sin embargo consigo 
describe también como sentimiento y aunque perteneciente a la eticidad lo hace solo en la forma de lo natural en la familia. ${ }^{8}$

En su estudio sobre las relaciones de Hegel con el romanticismo comenta Innerarity que: «El amor romántico no es otra cosa que la azarosa y fugaz pasión entre sujetos a los que no vincula todavía la relación moral del matrimonio y la familia; es la efusividad de un sentimiento interior, la pasión incapaz de darse a sí misma ninguna obligación. En la pasión, el amor no ha alcanzado todavía su concepto» (Innerarity 1993, p. 95), y en el final de la argumentación concluye que: «[Hegel] intenta armonizar el derecho y la pasión, a través de la idea de un deber que surge desde la libertad. Se trata de una libertad capaz de extenderse en el tiempo, más humana que la memoria sin libertad que caracteriza al derecho positivo, y más profunda que la libertad sin memoria de la pasión, tan encendida como fugaz. Solo quien no se haya rendido a la pobreza de una libertad inmediata puede ver en el compromiso y en el tiempo enemigos de la libertad» (Innerarity 1993, p. 103).

Parece claro que los conceptos de amor y amistad que Hegel conoce están teñidos del sentido que el romanticismo les dio en su momento. De ahí que algunos interpretes contemporáneos de la filosofía moral y política de Hegel

y no deje de mantenerse en lo universal. Este es el concepto concreto de la libertad, mientras que los dos momentos precedentes se han manifestado enteramente abstractos y unilaterales. Esta libertad la tenemos ya en la forma del sentimiento (in der Form der Empfindung), por ejemplo, en la amistad y en el amor (Freundschaft und Liebe). En estos casos el hombre no está unilateralmente dentro de sí, sino que se limita gustoso en relación con otro (beschränkt sich gern in Beziehung auf ein Anderes), pero en esta limitación se sabe como sí mismo. La libertad no radica, por lo tanto, en la indeterminación ni en la determinación, ella es ambas. La voluntad que se limita únicamente a un esto, es la voluntad del obstinado, que cree no ser libre si no tiene esa voluntad. Pero la voluntad no está atada a algo limitado sino que debe ir más allá, pues la naturaleza de la voluntad no es esa unilateralidad y esa dependencia, sino que la libertad es querer algo determinado y en esa determinación permanecer consigo y retornar nuevamente a lo universal», Hegel 2005, agregado al parágrafo 7.

8 «Amor (Liebe) significa conciencia de mi unidad con otro, de manera tal que no estoy para mí aislado, sino que consigo mi autoconciencia al abandonar mi ser por sí y saberme como unidad mía con el otro y como unidad del otro conmigo. Pero el amor es sentimiento (Empfindung), es decir, la eticidad en la forma de lo natural (die Sittlichkeit in Form des Natürlichen). En el Estado no existe ya esta forma, pues en él se es consciente de la unidad en la ley: su contenido debe ser racional y yo debo saberlo. El primer momento en el amor es que no quiero ser una persona independiente para mí y que si lo fuera me sentiría carente e incompleto. El segundo momento consiste en que me conquisto a mí mismo en otra persona y valgo en ella, lo cual le ocurre a esta a su vez en mí. El amor es por lo tanto una enorme contradicción (der ungeheuerste Widerspruch) que el entendimiento no puede resolver, pues no hay nada más consistente que esa puntualidad (Punktualität) de la autoconciencia que se niega y que sin embargo debo tener afirmativamente. El amor es al mismo tiempo la producción y la solución de la contradicción; en cuanto solución es la concordia ética (die sittliche Einigkeit)», Hegel 2005, par. 158 en el agregado. 
hayan dicho que: «La amistad en el sentido de la philia aristotélica no juega ningún papel en la filosofía práctica de Hegel, y ha pasado por alto cualquier otra noción del amor que no sea la romántica de unión como fusión.»»

En cuanto a la relación entre virtudes y polis, conviene decir que Hegel conoce la noción de hábito como correlato psicológico de cualquier virtud del carácter y la inteligencia. Habla del hábito como liberación de los sentimientos e impulsos ${ }^{10} \mathrm{y}$ también aunque en otro lugar lo hace de la virtud, a la que define como algo solo posible dentro de la esfera de la eticidad a no ser que se apele al heroísmo. ${ }^{11}$ Esto último es de importancia capital, y a juicio de Hegel ocurre por la diferencia esencial entre el modo de vivirse la eticidad en la polis griega y en el mundo moderno después del advenimiento del cristianismo. Ya que a partir de este ha surgido «el principio de la personalidad independiente y en sí misma infinita del individuo» (Hegel 2005, par. 185 y obs.), que debe ser respetado a partir de ese momento por el Estado.

Hegel considera el amor y la amistad como sentimientos al modo en que los interpretaron los románticos y por lo tanto como estadios previos a un desarrollo completo de la subjetividad como autoconciencia libre que solo se encuentra como en casa en el Estado como sistema de libertad y eticidad. Los momentos previos a ese desenlace son negados y conservados en el proceso dialéctico pero han de dejarse atrás por mor del estadio último. El mismo Hegel propor-

9 «Friendship, in the sense of Aristotle's philia, plays no role in Hegel's practical philosophy, and any conception of love other than the romantic one of fusion has escaped him», Peperzak 2001, pp. 648-649.

10 «Se suele hablar con desprecio del hábito [o costumbre], tomándolo como algo sin vida, contingente y particular. El contenido enteramente contingente es desde luego susceptible de recibir la forma del hábito, como cualquier otro contenido, y es el hábito de vivir lo que nos lleva a la muerte o, [dicho] de manera enteramente abstracta, es la muerte misma. Pero al mismo tiempo el hábito es lo más esencial de la existencia de toda espiritualidad en el sujeto individual, para que el sujeto, como inmediatez concreta, sea como idealidad anímica, para que el contenido religioso, moral, etc., le pertenezca a él en tanto es este sí mismo, en tanto es esta alma, y no esté en él como mero en sí (como disposición), ni como sensación transitoria o representación, ni tampoco como interioridad separada del hacer y de la realidad efectiva, sino que sea su ser», Hegel 1999, par. 410 y agregado.

11 «Lo ético, en tanto que se refleja en el carácter individual determinado como tal por la naturaleza, es la virtud. En la medida en que no muestra más que la adecuación del individuo a los deberes de las relaciones a las que pertenece, es la honradez (Rechtschaffenheit)» y en el agregado contonúa: En los estados antiguos, puesto que en ellos la eticidad no había llegado a ser este sistema libre que acoge en sí un desarrollo independiente y objetivo, era la genialidad propia del individuo la que tenía que llenar esta carencia. La doctrina de las virtudes, en la medida en que no sea una mera doctrina del deber y abarque lo particular del carácter basado en determinaciones naturales, se convierte de este modo en una historia natural del espíritu», Hegel 2005, par. 150. 
ciona las razones que hacen necesario este desarrollo (p.e. el descubrimiento de la conciencia subjetiva del individuo) de la polis griega al estado moderno.

\section{ARistóteles y Hegel están de ACUerdo en PARTE}

Una vez hemos llegado al apartado final del artículo queda por dilucidar la cuestión de hasta qué punto y en qué sentido Aristóteles y Hegel están o no de acuerdo.

A partir de la interpretación hecha en este texto de las relaciones entre ética y política no parece aventurado decir que tanto Hegel como Aristóteles reconocen la necesidad de que las instituciones políticas sirvan como marco para la adquisición y práctica de las virtudes. En el caso concreto de la philia al ser una virtud per se social y comunitaria la polis es el locus en el cual se dan las condiciones necesarias para que se produzca ese tipo de relación interpersonal. Ahora bien mientras que Hegel considera que la amistad per se no es una de las figuras o momentos necesarios de la eticidad - lo son la familia, la sociedad civil y el Estado - sino que permanece encerrada en el terreno de la moralidad, para Aristóteles la philia politike constituye el fin que han de buscar los legisladores y gobernantes como un bien intrínseco de la mejor polis y del carácter del buen ciudadano.

Para Aristóteles hay una conexión necesaria entre el fin del hombre y de la polis, en ambos casos la eudaimonia y la philia son necesarias, como lo es en definitiva la práctica de las virtudes. De esto sí que se hace eco Hegel en parte cuando interpreta la filosofía práctica de Aristóteles como una relación intrínseca entre ética y política, por ejemplo en los siguientes pasajes de sus Lecciones de historia de la filosofía dedicados a la filosofía práctica del estagirita:

«Aristóteles se dio cuenta, más o menos claramente, de que la sustancia positiva, la necesaria organización y realización del espíritu práctico es el Estado, que es realizado por medio de la actividad subjetiva de tal modo que esta encuentra en él su determinación. Por eso también ve en la filosofía política toda la filosofía práctica y el fin del Estado como la felicidad general», (Hegel 1985, p. 315). «Como se ve, Aristóteles no considera como lo primario al individuo y sus derechos, sino que reconoce al Estado como lo superior en cuanto a su esencia, como algo más alto que el individuo y la familia, ya que es aquél precisamente el que constituye la sustancialidad de estos», (Hegel 1985, p. 316).

No obstante, la omisión de un tratamiento pormenorizado de la philia y de sus repercusiones para el desarrollo del individuo y el todo social hacen poco aristotélico el acercamiento hegeliano. Como se ha intentado hacer ver, son varias las razones que se pueden aducir para dar sentido a esta carencia. A esto se podría objetar al menos de dos maneras: (1) que Hegel no hace sino desarrollar y completar lo que estaba implícito en la teoría aristotélica, como 
defiende Goldstein; o que como afirma Williams (2) dicho tratamiento sí que se encuentre en la teoría hegeliana pero solo de modo latente.

La primera objeción la he tomado del trabajo de Goldstein: Hegel's Idea of the Good Life. A juicio de Goldstein: «las virtudes morales aristotélicas en último término responden a la demanda que la polis pide a sus ciudadanos para asegurar la perpetuación de la ciudad en su naturaleza específica. Cuando Hegel define la virtud como la reflexión ética del carácter, captura la virtud como uno de los requerimiento de la polis como esto ya se dan en el espíritu humano. En la especificación, en la forma de la honestidad, de esta adecuación del espíritu humano a los requerimientos particulares de la comunidad se completa y preserva lo esencia de la virtud moral de los griegos» (Goldstein 2006, p. 221).

No osbtante como ya hemos visto, y como afirma Strauss, para Aristóteles es más bien la polis la que está subordinada al ejercicio de las virtudes y no ocurre al revés como defiende Hegel.

La segunda objeción la he extraído del artículo de Williams: «Aristotle, Hegel, and Nietzsche on Friendship». Williams cree ver una conexión esencial en las propuestas aristotélica y hegeliana. Este interpreta que la philia tal y como es entendida por Aristóteles puede relacionarse de manera directa al modo en que se relacionan dos subjetividades que se reconocen mutuamente como seres libres y autoconscientes, ${ }^{12}$ aunque matiza que: «el tratamiento aristotélico de la philia tiene el defecto de ser inmediata, provinciana y abstracta» (Williams 2012, p. 68).

Aunque de hecho Aristóteles pone como una de las condiciones para hablar de philia entre dos individuos que estos se reconozcan mutuamente como amigos, también es cierto que dicho reconocimiento no funda la amistad. Más bien es el carácter excelente del amigo y lo que se deriva de éste y del propio (p.e., el buscar $s u$ bien antes que el propio) lo que establece una amistad plena en sentido aristotélico. Por otra parte, la philia politike que se da entre conciudadanos en la mejor polis también subordina la relación de reconocimiento mutuo a la búsqueda del bien y la práctica de las virtudes.

En definitiva y por formularlo en modo interrogativo: ¿Bastaría con que Hegel reconozca la necesidad de las virtudes para el desarrollo del individuo y del Estado para mantener una continuación y no una desfiguración de la filosofía práctica de Aristóteles? A mi juicio, la pérdida de la philia por el camino es una carencia de la propuesta hegeliana siempre y cuando esta quisiera ser una continuación de la especulación aristotélica.

12 «My thesis is that reciprocal recognition is the deep structure of intersubjectivity inherent in spirit and Sittlichkeit. I hope to show not only that recirpocal recognition in Hegel's sense is the deep intersubjective structure of Friendship -the intersubjective tie that binds- but also that Aristotle's account of Friendship exhibits mutual recognition», Williams 2012, p. 55. 
Quizá una de las razones de esta discontinuidad sea que a diferencia de Aristóteles que considera de modo teleológico el proceso que conduce a la polis desde la familia, por su parte Hegel lo hace de modo dialéctico. Esto repercute de manera directa sobre la superación del amor y la amistad en el estadio ético definitivo que es el Estado, mientras que para Aristóteles es una forma actualizada de la philia la que surge con el advenimiento de la polis.

\section{BIBLIOGRAFÍA}

AQUINATIS, Thomae, S., In decem libros Ethicorum Aristotelis Ad Nichomachum expositio, Italia: Marietti, 1964.

ARISTÓTELES, Ética a Nicómaco, edición bilingüe y traducción de María Araujo y Julián Marías. Introducción y notas de Julián Marías, Madrid: Centro de Estudios Políticos y Constitucionales, 2014.

Política, edición bilingüe y traducción de Julián Marías y María Araujo. Introducción y notas de Julián Marías, Madrid: Centro de Estudios Políticos y Constitucionales, 2005.

Retórica, introducción, traducción y notas de Alberto Bernabé Pajares, Madrid: Alianza editorial, 2000.

AUBENQUE, P., La prudencia en Aristóteles. Con un apéndice de la prudencia en Kant, Barcelona: Crítica, 1999.

BARKER, E., The Politics of Aristotle, Oxford: Oxford Clarendon Press, 1968.

COOPER, John, M., «Aristotle's Concept of Friendship» en Essays on Aristotle's Ethics, ed. A. O. Rorty, Berkeley: University of California Press, 1980, 301-340.

FERRARIN, A., Hegel and Aristotle, New York: Cambridge University Press, 2001.

GOLDSTEIN, Joshua, D., Hegel's Idea of the Good Life. From Virtue to Freedom, Early Writings and Mature Political Philosophy, Dordrecht: Springer Netherlands, 2006.

HEGEL, G. W. F., Principios de la filosofía del derecho o Derecho natural y ciencia política, Barcelona: Edhasa, 2005.

Escritos de juventud, México: FCE, 1984.

Lecciones de filosofía de la historia, Barcelona: PPU, 1989.

Lecciones sobre la historia de la filosofía (II), México: FCE, 1985.

Fenomenología del Espíritu, (ed. bilingüe), Madrid: Adaba Editores/ UAM Ediciones, 2010.

Enciclopedia de las ciencias filosóficas, Madrid: Alianza Editorial, 1999.

INNERARITY, D., Hegel y el romanticismo, Madrid: Tecnos, 1993.

INWOOD, M., The Blackwell Philosopher Dictionaries. A Hegel Dictionary, Oxford: Blackwell Publishers, 2003. 
Amistad y reconocimiento. Sobre la philia aristotélica...

JAEGER, W., Aristóteles. Bases para la historia de su desarrollo intelectual, México: FCE, 2000.

KONSTAN, D., «Aristotle on Love and Friendship» en $\Sigma$ XO $\Lambda$ H vol. II. 2 (2008), 207-212.

PEPERZAK, Adriaan, T., Modern Freedom. Hegel's legal, Moral and Political Philosophy, Dordrecht-Boston-London: Kluwer Academic Publishers, 2001.

POLO, L., «La amistad en Aristóteles» en Anuario Filosófico 1999 (32), 477-485.

Persona y libertad, Pamplona: Eunsa, 2007.

STRAUSS, L., CROPSEY, J., (eds.) History of Political Philosophy, Chicago: Chicago University Press, 1987.

STRAUSS, L., Natural Right and History, Chicago: Chicago University Press, 1965.

The City and Man, Chicago: Chicago University Press, 1978.

VÁZQUEZ DE PRADA, A., Estudio sobre la amistad, Madrid: Rialp, 1975.

VOEGELIN, E., Order and History (III. Plato and Aristotle), Baton Rouge: Louisiana State University Press, 1957.

La nueva ciencia de la política. Una introducción, Buenos Aires: Katz, 2006.

WIELAND, W., «Norma y situación en la ética aristotélica» en Anuario filosófico 1999 (32), 107-127

WILliAMS, Robert, R., Tragedy, Recognition, and the Death of God. Studies in Hegel and Nietzsche, Oxford: Oxford University Press, 2012.

ZAGAL, H., Amistad y felicidad en Aristóteles, México: Ariel, 2014.

Miguel Martí SÁnchez es doctor en filosofía.

Lineas de investigación:

Filosofía Antigua, especialmente la filosofía teórica y práctica de Aristóteles. Historia de la metafísica, Fenomenología, Antropología filosófica y ética.

Publicaciones recientes:

«¿Cuál es el método de la Ética?» en Auctoritas prudentium. Revista de la Facultad de Derecho de la Universidad del Istmo (Guatemala), ISSN 2305-9729, Año VIII (2016), No. 14, 5-19.

«La filosofía de las matemáticas de Aristóteles», en Tópicos. Revista de Filosofía (52) 2017, 43-66.

«Homo capax Dei. La posibilidad del conocimiento teórico de Dios según L. Polo», en Studia Poliana (19) 2017, 27-40.

Email:mmarti.1@alumni.unav.es 
